\begin{tabular}{|c|l|}
\hline Title & $\begin{array}{l}\text { Fiber-microsphere laser with a submicrometer sol-gel silica glass layer codoped with erbium, al uminum, and } \\
\text { phosphorus }\end{array}$ \\
\hline Author(s) & Takashima, Hideaki; Fujiwara, Hideki; Takeuchi, Shigeki; Sasaki, Keiji; Takahashi, Masahide \\
\hline Citation & $\begin{array}{l}\text { A pplied Physics Letters, 90(10), 101103 } \\
\text { https://doi.org/10.1063/2711384 }\end{array}$ \\
\hline Issue Date & 2007-03-05 \\
\hline Doc URL & http://hdl.handle.net/2115/33857 \\
\hline Rights & $\begin{array}{l}\text { Copyright 2007 A merican Institute of Physics. This article may be downloaded for personal use only. A ny other use } \\
\text { requires prior permission of the author and the A merican Institute of Physics. }\end{array}$ \\
\hline Type & article \\
\hline File Information & A pplPhysLett_90_101103.pdf \\
\hline
\end{tabular}

Instructions for use 


\title{
Fiber-microsphere laser with a submicrometer sol-gel silica glass layer codoped with erbium, aluminum, and phosphorus
}

\author{
Hideaki Takashima, Hideki Fujiwara, Shigeki Takeuchi, ${ }^{a)}$ and Keiji Sasaki \\ Research Institute for Electronic Science, Hokkaido University, Sapporo 060-0812, Japan \\ Masahide Takahashi \\ Institute for Chemical Research, Kyoto University, Uji, Kyoto 611-0011, Japan
}

(Received 6 October 2006; accepted 29 January 2007; published online 5 March 2007)

\begin{abstract}
Lasing of a taper-microsphere system with a gain layer of submicrometer thickness is demonstrated. For the gain layer, an $\mathrm{Er}^{3+}$-doped $\mathrm{P}_{2} \mathrm{O}_{5}-\mathrm{Al}_{2} \mathrm{O}_{3}-\mathrm{SiO}_{2}$ thin film with a thickness of $200 \mathrm{~nm}$ was fabricated using the sol-gel method on a silica microsphere. The demonstration of single-mode lasing with the thin gain layer suggests the improved dispersion of Er ions in the P codoped gain layer. (C) 2007 American Institute of Physics. [DOI: 10.1063/1.2711384]
\end{abstract}

Microsphere resonators coupled with a tapered optical fiber have been attracting considerable attention recently as ideal cavity systems having ultrahigh $Q$ factors and single spatial mode input-outputs. After pioneering demonstrations of coupling between a silica glass microsphere and a tapered fiber, ${ }^{1,2}$ demonstrations of lasing phenomena have been reported. $^{3-8}$ One concern has been to improve the coupling parameter $(\beta)$ between the spontaneous emission of the gain medium and the cavity mode in the microsphere in order to realize ultralow threshold lasers. ${ }^{9}$ Realization of a submicrometer thick gain layer is essential for achieving better coupling between the gain layer and a cavity mode in the microsphere, since the spatial distribution of the mode is about $1 \mu \mathrm{m}$.

The first demonstration of a fiber-microsphere laser was reported by Cai et al. using an erbium:ytterbium-codoped phosphate glass microsphere. ${ }^{3}$ Later, demonstrations using tellurite glass microspheres with doped $\mathrm{Er}$ ions ${ }^{4}$ and with doped Tm ions ${ }^{5}$ were also reported. In those experiments, however, the whole spheres were filled with the gain media. Yang and Vahala applied erbium-doped sol-gel films to the surface of a silica microsphere. ${ }^{6}$ The thickness of the gain region was varied from $1 \mu \mathrm{m}$ to more than $5 \mu \mathrm{m}$. They observed continuous wave operation for microspheres having thin gain layers $(1 \mu \mathrm{m})$, while pulse mode operation was observed in microspheres having thicker $(\geqslant 5 \mu \mathrm{m})$ gain layers. Recently, Hoi et al. adopted erbium-doped sol-gel silicaalumina glasses as the gain layer. ${ }^{7}$ They succeeded in increasing the concentration of Er ions upto 15000 ppm by aluminum codoping. However, the thickness of the gain medium was still more than $1 \mu \mathrm{m}$. In earlier studies, ${ }^{6,7}$ the spheres were repeatedly dipped into the coating sol, heated and irradiated by a laser beam even for gain layers having a $1 \mu \mathrm{m}$ thickness since the buildup rate was $0.3 \mu \mathrm{m} /$ cycle. $^{6}$ Recently, an ion implantation technique was used to control the position and thickness of the gain region; ${ }^{8}$ however, an ion accelerator is required for such a process.

In this letter, we report single-mode lasing of a tapermicrosphere system having a gain layer of a thickness of a few hundred nanometers using phosphorus codoped sol-gel erbium silica-aluminum glass. Our trial of phosphorus dop-

${ }^{a)}$ Electronic mail: takeuchi@es.hokudai.ac.jp ing is motivated by a recent electron spin resonance study ${ }^{10}$ that reported a striking difference between $\mathrm{Al}$ and $\mathrm{P}$ codopings for the local structure near Er ions, suggesting a better dispersion of highly doped $\mathrm{Er}$ ions with $\mathrm{P}$ codoping. We consider that the improved dispersion of $\mathrm{Er}$ ions in our $\mathrm{P}$ codoped gain layer resulted in the realization of the lasing using a gain layer a few hundred nanometers thick. It should be noted that the gain layer was formed by performing dipping, heating, and irradiating with a laser irradiation once; repetition of the coating processes was not necessary for our thin gain layers.

The coating sol was prepared using ethanol (EtOH), dimethylformamide (DMF), tetraethoxysilane (TEOS), hydrochloric acid $(\mathrm{HCl})$, aluminum chloride $\left(\mathrm{AlCl}_{3}\right)$, eribum chloride hexahydrate $\left(\mathrm{ErCl}_{3} \cdot 6 \mathrm{H}_{2} \mathrm{O}\right)$, and triethylphosphate $\left(\mathrm{C}_{2} \mathrm{H}_{5} \mathrm{O}\right)_{3} \mathrm{PO}$ as follows. A mixture of EtOH, DMF, TEOS, and $\mathrm{HCl}$ was stirred at $0{ }^{\circ} \mathrm{C}$ for $1 \mathrm{~h}$. At this temperature, $\mathrm{AlCl}_{3}$ dissolved in $\mathrm{EtOH}$ was added to the solution, and it was stirred for another $1 \mathrm{~h}$. Then, $\mathrm{ErCl}_{3} \cdot 6 \mathrm{H}_{2} \mathrm{O}$ dissolved in $\mathrm{EtOH}$ was mixed in the same way for $\mathrm{AlCl}_{3}$. After this, distilled water was slowly added to the solution, and it was then stirred at room temperature for $17 \mathrm{~h}$. This solution was used as the coating sol for reference samples that did not have phosphorus. After stirring, $\left(\mathrm{C}_{2} \mathrm{H}_{5} \mathrm{O}\right)_{3} \mathrm{PO}$ was added to this solution and was stirred for another $12 \mathrm{~h}$. The obtained coating sol was used to form $\mathrm{Er}^{3+}$-doped $\mathrm{P}_{2} \mathrm{O}_{5}-\mathrm{Al}_{2} \mathrm{O}_{3}-\mathrm{SiO}_{2}$ films.

Silica microspheres with stems were formed by melting the edge of a tapered fiber tip using radiation from a $\mathrm{CO}_{2}$ laser. $^{11,12}$ The diameters of the microspheres used were from about 40 to $200 \mu \mathrm{m}$. The microspheres were immersed in the coating sol for $30 \mathrm{~s}$ with the stem attached to a motorized linear stage. After withdrawing the microspheres at a speed of $3 \mathrm{~cm} / \mathrm{min}$ from the solution, the microspheres were kept with their spheres pointing up (i.e., their stems pointing down) to avoid extra accumulation of the coating sol on the surface of the spheres. The microspheres were then annealed in an electrical furnace at $900{ }^{\circ} \mathrm{C}$ for $10 \mathrm{~min}$. Then, the microspheres were irradiated with a $\mathrm{CO}_{2}$ laser for a few tens of seconds to anneal out microcracking in the sol-gel surface. ${ }^{6}$ The molar composition of the fabricated films was $\mathrm{SiO}_{2}: \mathrm{Al}_{2} \mathrm{O}_{3}: \mathrm{P}_{2} \mathrm{O}_{5}: \mathrm{Er}_{2} \mathrm{O}_{3}=1: 0.12: 0.05: 0.01$, corresponding to a concentration of $\mathrm{Er}$ ions of $10^{4} \mathrm{ppm}$. For reference samples having thicker gain layers, the above-mentioned 


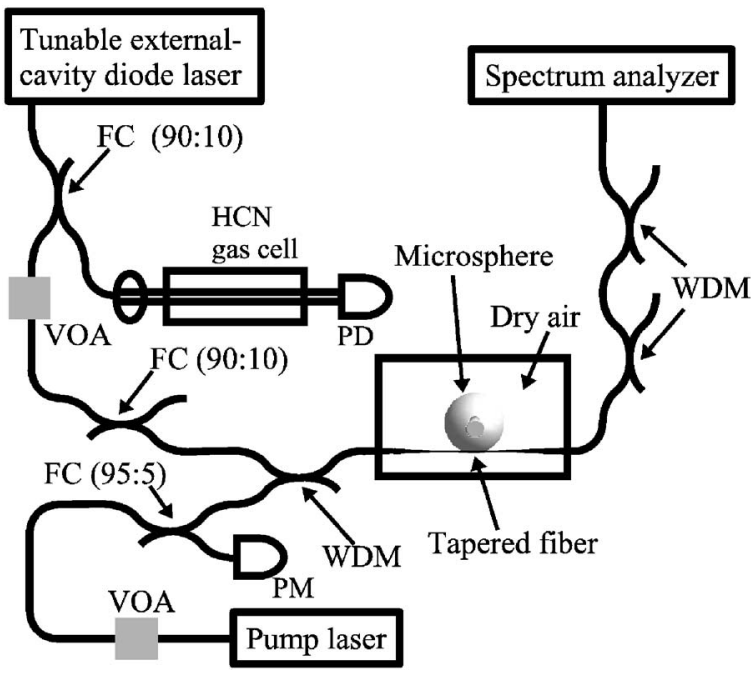

FIG. 1. Schematic of experimental setup. VOA: variable optical attenuator, FC: $95 / 5$ fused fiber coupler, PM: optical power meter, and WDM: 980/1550 nm wavelength division multiplexer.

coating (dipping and annealing) process was repeated for the required number of times, followed by laser irradiation. The thicknesses of the sol-gel films fabricated on the surface oxidized silicon substrate using the same procedures were analyzed using an ellipsometer. The thickness of the sol-gel film having a single coating was estimated to be $200 \mathrm{~nm}$, which is consistent with the measurement result of $570 \mathrm{~nm}$ for a film having a triple coating and comparable with a previously reported buildup rate of $\sim 0.3 \mu \mathrm{m} /$ cycle. $^{6}$ We consider that the thicknesses of the films on the silica microsphere are almost same as these values.

A tapered fiber (diameter $2.75 \pm 0.25 \mu \mathrm{m}$ ) was fabricated by heating a single mode fiber (Thorlabs, 1060XP) with a ceramic heater and stretching the end of the fiber. ${ }^{13,14}$ The transmittance of the fiber was about $90 \%$ (when fablicated) and flat for the wavelength region from 1500 to $1620 \mathrm{~nm}$, indicating single mode operation at $1550 \mathrm{~nm} .{ }^{14}$

The experimental setup for acquiring the lasing spectrum is shown in Fig. 1. A single-mode laser diode operating at 975 nm (Thorlabs, PL980P200) with single-mode optical fiber output was used for pumping. The intensity of the pump light was controlled using a variable optical attenuator. The intensity of the pump power was monitored using an optical power meter (Newport, 2835-C) connected to the output of an unbalanced (95:5) fused fiber coupler. A probe light of a tunable single-frequency diode laser (wavelength 1500 to $1620 \mathrm{~nm}$, Santec TSL-210V) was coupled via a 980/1550 nm wavelength division multiplexer (WDM) in order to check the coupling of the tapered fiber and the microsphere. The output of the WDM was connected to the tapered fiber. The probe light was turned off when during the lasing experiments.

One of the prepared microsphere samples was gently touched to the tapered fiber under microscope observation using a two-dimensional piezomanipulator, which gripped the stem of the sphere. The microsphere and the tapered fiber were in a plastic box, which was filled with dry air. ${ }^{13}$ Unabsorbed pump light $(975 \mathrm{~nm})$ was removed from the laser emission light (about $1550 \mathrm{~nm}$ ) using two 980/1550 nm WDM couplers. Then, the laser emission light was analyzed

using an optical spectrum analyzer (Anritsu, MS9001A). FIG. 3. Laser output power vs pump power at $975 \mathrm{~nm}$.
Downloaded 22 Apr 2008 to 133.87.26.199. Redistribution subject to AIP license or copyright; see http://apl.aip.org/apl/copyright.jsp

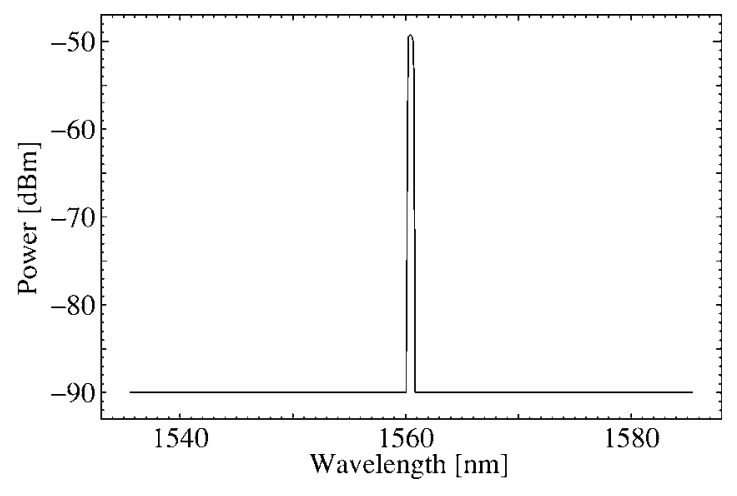

FIG. 2. Single-mode lasing spectrum of a microsphere having a diameter of $38 \pm 1 \mu \mathrm{m}$.

Note that the output powers shown in Figs. 2 and 3 are estimated values at the end of the tapered fiber obtained by compensating for optical losses $(19.5 \%)$ at the two WDM couplers and the fiber connectors.

Figure 2 shows a single-mode lasing spectrum of a microsphere (diameter of $38 \pm 1 \mu \mathrm{m}$ ) having a 200-nm-thick P-doped gain layer produced using a single coating process. The pump power launched into the tapered fiber was $670 \mu \mathrm{W}$, which is an estimated value for the input of the tapered fiber after the WDM (Fig. 1) measured using the monitor power meter (PM in Fig. 1). The spectrum shown was measured at a resolution of $0.5 \mathrm{~nm}$. Even when we used a higher resolution of $0.1 \mathrm{~nm}$, the single peak was not discriminated. During the experiment, the microsphere was in contact with the tapered fiber. The wavelength of the pump laser was fine tuned by adjusting the temperature of the laser diode to give the highest output signal. When we adjusted the contact position of the tapered fiber on the microsphere, we observed both single-mode and multimode lasings (not shown), but this behavior was not dependent on the power or the wavelength of the pump laser.

Figure 3 shows the output power versus the pump power launched into the tapered fiber for the same sample and the same conditions as those for Fig. 2. Again, the pump powers are estimated values at the input of the tapered fiber, and the output power is the peak value of the single-mode lasing spectrum (Fig. 2). Above a threshold input pump power, the laser output power increased linearly with the pump power. The threshold was estimated to be about $180 \mu \mathrm{W}$ by fitting the data with a linear function. This threshold value is comparable to that found in a previous study. ${ }^{6}$

We tested another microsphere having a diameter of $60 \mu \mathrm{m}$ and a gain layer having a thickness estimated to be

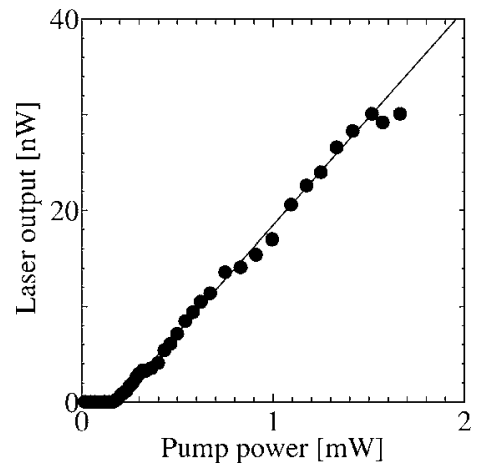

FIG. 3. Laser output power vs pump power at $975 \mathrm{~nm}$. 5 
$560 \mathrm{~nm}$, produced using a multiple coating process. We also observed single-mode lasing for low pump powers in this sample. However, for this sample, single-mode lasing became multimode lasing when the pump power was increased.

We also tried to observe lasing in microspheres that did not have $\mathrm{P}$-doped $\left(\mathrm{Er}^{3+}\right.$-doped $\left.\mathrm{Al}_{2} \mathrm{O}_{3}-\mathrm{SiO}_{2}\right)$ gain layers using several samples (diameters of 45 to $150 \mu \mathrm{m}$ and gain layer thicknesses estimated to be 70 to $510 \mathrm{~nm}$ ), including a sample (diameter of $45 \mu \mathrm{m}$ and gain layer thickness of $280 \mathrm{~nm}$ ) whose size and gain-layer thickness were similar to the one used to obtain the data shown in Figs. 2 and 3. However, we were not able to observe lasing (neither single mode nor multimode) for those P-undoped samples.

According to a recent electron spin resonance study, ${ }^{10}$ there is a striking difference between $\mathrm{Al}$ and $\mathrm{P}$ codopings for the local structure near Er ions; doped P ions preferentially coordinate to the Er ions to form a "solvent shell structure" which results in a better dispersion of highly doped Er ions. We think the improved dispersion of Er ions in our phosphorus codoped gain layer reduced cross relaxation of $\mathrm{Er}$ ions and produced a higher efficiency, resulting in the realization of the lasing in a gain layer which is a few hundred nanometers thick.

In conclusion, we have reported single-mode lasing of a taper-microsphere system having a gain layer of a few hundred nanometers thick using $\mathrm{P}$ codoped sol-gel Er silicaaluminum glass. We consider that the improved dispersion of Er ions in our P codoped gain layer resulted in the demonstration of the lasing in a gain layer, which was a few hundred nanometers thick. In our experiment, the gain layer was formed using a single coating process.

Numerical calculation based on the Mie scattering theory suggests that the intensity of the resonant-mode field near the surface, where the gain layer of the current sample exists, is just $20 \%$ of the highest intensity at about $600-800 \mathrm{~nm}$ inside from the surface. Thus, the coupling between the gain layer and the resonant mode will be much improved when the gain layer is adjusted to this position.
The control of the gain layer position may be possible by overcoating of undoped sol-gel silica glass on the precoated gain layer. Although it is difficult to estimate the laser performance precisely, the $\beta$ parameter will be much improved resulting in the significant decrease of the laser threshold.

The authors would like to thank Eun-Seok Kang and Hidenori Konishi for technical assistance. The current work was partly supported by the program "R\&D support scheme for funding selected IT proposals" of the Ministry of Public Management, Home Affairs, Posts and Telecommunications, a Grant-in-Aid from the Japan Society for the Promotion of Science, the 21st Century COE program, CREST-Project and PRESTO-Project of the Japan Science and Technology Agency.

${ }^{1}$ J. C. Knight, G. Cheung, F. Jacques, and T. A. Birks, Opt. Lett. 22, 1129 (1997).

${ }^{2}$ M. Cai and K. Vahala, Opt. Lett. 25, 260 (2000).

${ }^{3}$ M. Cai, O. Painter, and K. J. Vahala, Opt. Lett. 25, 1430 (2000).

${ }^{4}$ X. Peng, F. Song, S. Jiang, N. Peyghambarian, M. Kuwata-Gonokami, and L. Xu, Appl. Phys. Lett. 82, 1497 (2003).

${ }^{5}$ K. Sasagawa, Z. Yonezawa, R. Iwai, J. Ohta, and M. Nunoshita, Appl. Phys. Lett. 85, 4325 (2004).

${ }^{6}$ L. Yang and K. J. Vahala, Opt. Lett. 28, 592 (2003).

${ }^{7}$ P. V. Hoi, C. T. T. Ha, and H. Q. Hung, Appl. Phys. Lett. 87, 161110 (2005).

${ }^{8}$ J. Kalkman, A. Polman, T. J. Kippenberg, K. J. Vahala and M. L. Brongersma, Nucl. Instrum. Methods Phys. Res. B 242, 182 (2006).

${ }^{9}$ R. J. Horowicz, H. Heitmann, Y. Kadota, and Y. Yamamoto, Appl. Phys. Lett. 61, 393 (1992).

${ }^{10}$ A. Saitoh, S. Matsuishi, C. Se-Weon, J. Nishii, M. Oto, M. Hirano, and H. Hosono, J. Phys. Chem. B 110, 7617 (2006).

${ }^{11}$ A. Chiba, H. Fujiwara, J. Hotta, S. Takeuchi, and K. Sasaki, Jpn. J. Appl. Phys., Part 1 28, 6138 (2004).

${ }^{12}$ H. Takashima, H. Fujiwara, J. Hotta, S. Takeuchi, K. Sasaki, S. Murakami, T. Torimoto, and B. Ohtani, Jpn. J. Appl. Phys., Part 1 45, 6917 (2006).

${ }^{13}$ A. Chiba, H. Fujiwara, J. Hotta, S. Takeuchi, and K. Sasaki, Appl. Phys. Lett. 86, 261106 (2005).

${ }^{14}$ H. Konishi, H. Fujiwara, S. Takeuchi, and K. Sasaki, Appl. Phys. Lett. 89, 121107 (2006). 